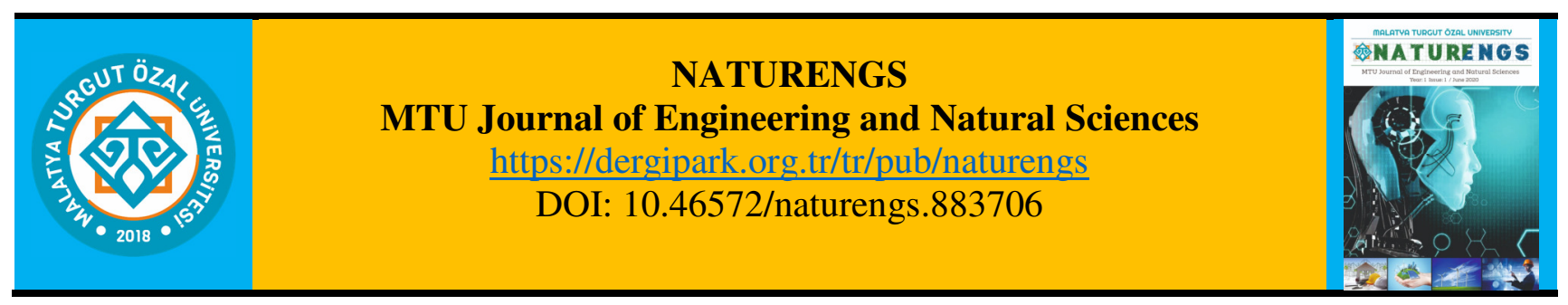

Review Article

\title{
Microfluidic Technology and Biomedical Field
}

\author{
Zülfü TÜYLEK \\ Electronics and Automation Department, Yesilyurt Vocational School, Malatya Turgut Ozal University, \\ Malatya, Turkey.
}

(Received: 20.02.2021; Accepted: 21.05.2021)

\begin{abstract}
It is seen that the development of microfluidic laboratories working passively on chips has increased over the years. The field of microfluidics includes the use of microstructured devices, which typically have micrometer sizes and allow precise processing of low volumes. Nano fields are the main fields of nanotechnology, which includes science fields such as earth science, organic chemistry, molecular biology, semiconductor physics, micromachinery where the control of the atomic and molecular unit will take place. New techniques are needed to meet existing for the development phase. Micro and nano-volume multi-stage systems through micrometer-sized channels and microfluidics, which are applied science branches, have drawn significant attention in engineering. The circulation of fluids through micrometer-sized channels examines factors that can affect the behavior of fluids, such as surface tension, the utilization of energy, and fluid resistance in the system. Microfluidic devices and systems have a variety of functions to replace routine biomedical analysis and diagnostics. It emphasizes a higher level of system integration with advanced automation, control and High-Efficiency processing potential while consuming small amounts of sample and reagent in less time. Thanks to miniaturization, better diagnostic speed, cost-effectiveness, ergonomics and sensitivity are achieved.

This work presents a detailed literature survey on the field of microfluidic technology, including system components. There are two main objectives of the review study: First, to provide the mechanisms, applications and recent developments on microfluidic techniques and Second, to suggest current research topics and possible future research in the biomedical field in microfluidic technology.
\end{abstract}

Keywords: Microfluidic, Microtechnology, Miniaturized, Sensors, SARS-CoV-2.

\section{INTRODUCTION}

It is very important to use small volumes of fluids in high volume scanning, diagnostic and research applications. Microfluidics is a way of processing small volume liquids between microlitres and picolitres. Existing literature reports various aspects of these devices including fluid transfer, system characteristics, detection techniques, and bioanalytical applications. Low production costs, cost-effective disposable chips and allowing mass production are reasons for a preference [1]. In the Hagen-Poiseuille studies in the 1840s, it is seen that they did the study related to the chemical structure of the polidimetilsiloksan (PDMS) material used in microfluidics. Dow Corning founded Midland in 1943 to work on silicones and is its first manufacturer. In the 1950s, thanks to advances in semiconductor technology, the design of miniature systems and components began. In 1958 Jack Kilby started working with the first microchannels [2]. In a study by Stephen Terry in the mid-1970s at Stanford University, a miniature gas chromatograph in the form of a wafer was produced by superimposing silicon sheets. Gas chromatography creates the first working example to be called a laboratory on a 
chip (lab on a chip) that creates a miniature thermal conductivity detector as capillary path inputs and outputs through the sample injection system. In 1993, a glass chip was developed to perform capillary electrophoresis of amino acids in a matter of seconds. With help of this work, it is possible to create a miniature laboratory on a chip that can be used for complex analysis.

Nowadays, researchers have conducted studies to develop fast passive labs on chips to meet the demands of the biomedical field. Recent developments indicate that passive microfluidic techniques including mechanisms and applications considerably increase. Microfluidics is used for various laboratory experiments such as drug testing and discovery, filtration and particle separation, cell separation and counting, cell culture, 3D printing, stoichiometry, and flow synthesis [3].

\section{FUNCTIONALITY IN MICROFLUIDIC DEVICES}

Microfluidic techniques, related mechanisms and applications are being developed day by day. It will be possible to list the microfluidic components used today under the following headings.

\subsection{Cell Manipulation}

Two cells in a genetically identical group are different from each other. Cell heterogeneity plays an important role in the accurate interpretation of diagnostic and therapeutic consequences of diseases. Therefore, various cell manipulation techniques have been developed for specific purposes and applications in microfluidics. Cell manipulation techniques in microfluidics could be categorized as externally applied forces for optical, magnetic, electrical, mechanical, and other manipulations [4]. The use of immobile microfluidics without the external force among cell manipulation techniques has attracted great interest. Recently, microfluidic systems used are suitable for simple and highly efficient cell separation and analysis [5]. Silicone is the first choice among microfluidic system manufacturing when considering advantages such as its readiness, chemical compatibility and thermostability. Ease of manufacture, design flexibility, semiconductor properties, and the possibility of surface modifications are sufficient reasons why silicon has been the dominant material for microfluidic platforms for decades [6].

\subsection{Cell, Tissue and Organ Culture Platforms}

The emerging technology of microfluidic cell culture systems has the potential to make a significant impact on cell biology research. Many cells in our body do not participate in the circulation, instead, they depend on an environment called an extracellular matrix to survive. For example, proteins called integrin receive specific signals as a result of the physical attachment of cells to the matrix and focal adhesion sites and transmit them to the skeletal machine inside the cell. Therefore, skeletal proteins for many cells, including integrin, exist in the 3-dimensional physical microenvironment [7]. A complex three-dimensional extracellular (in vivo) matrix causes radical changes in cell morphology and function. Miniature culture systems (in vivo) have improved tissue functions compared to existing in vivo studies. This allows the complex mechanisms underlying tissue growth, regeneration and disease to be studied more accurately than existing in vitro models, without the inherent difficulties of in vivo studies [8]. Luo et al. studied the numerical simulation of oxygen and glucose transport to improve the functionality of biomedical microfluidic devices. They designed an integrated microfluidic device for the culture of individual coral polyps, including a uniform flow medium, rapid mass transfer, and precise temperature control [9]. The microfluidic device provided a reliable analytical approach for model and mechanism studies of coral bleaching and reef 
conservation. Williams et al. addressed one of the problems with microfluidic cell culture platforms and found that the confinement of unwanted air bubbles in the gate often caused cell damage or device delamination [10].

\subsection{Sensors}

The foundations of the microfluidic industry were laid in the early 1990s and gained momentum with the development of microtechnology. It is a multidisciplinary structure combining the fields of physics, chemistry, biology, engineering, nanotechnology, medicine and biotechnology. By creating micro-channels in a material, it enables biological fluids, even biological particles such as cells and organisms, to be prepared for analysis at a certain speed. Horiuchi et al. It is seen that he developed an immunoassay chip consisting of vertically integrated capillary tubes to create negative pressure and pump the liquid towards it [11]. The Lab on a Chip (LOC) concept is a system that combines fluid mechanics, physics and nanotechnology in which large laboratory tests are reduced to a single stage. On-chip sensors integrated with microfluidic devices have great potential in the On-chip laboratory or independent systems for various biological and biomedical applications [12]. The flow of the surface tension in the liquid through the microchannels is triggered due to the change in liquid volumes. Passive microfluidics with the electrochemical sensor in the microchannel is suitable for LOC flow injection. Basic principles of nano and microfluidic chips are detection of RNA / DNA from target organisms or antigen and toxin substances specific to target organisms [13]. This detection takes place through biosensors. Biosensors are a transducer device that detects the presence of substances by binding the substances to be analyzed and interacts with the sensor and can signal this interaction. The use of nanomaterials in the design of biological sensors; enables the process to be carried out in a short time, to be portable and to be processed at low cost. Therefore, nanotechnology tools provide a broader perspective on new vaccine design strategies. For example, a nano-based formulation for SARS-CoV-2 therapeutics is being developed as a delivery tool, along with a new nano vaccine metastasis platform and nano drugs useful for the treatment of SARS-CoV-2 infections. Scientists are now working to quickly identify and develop appropriate nano-vaccines and treatment options, including new nanobased technologies [14].

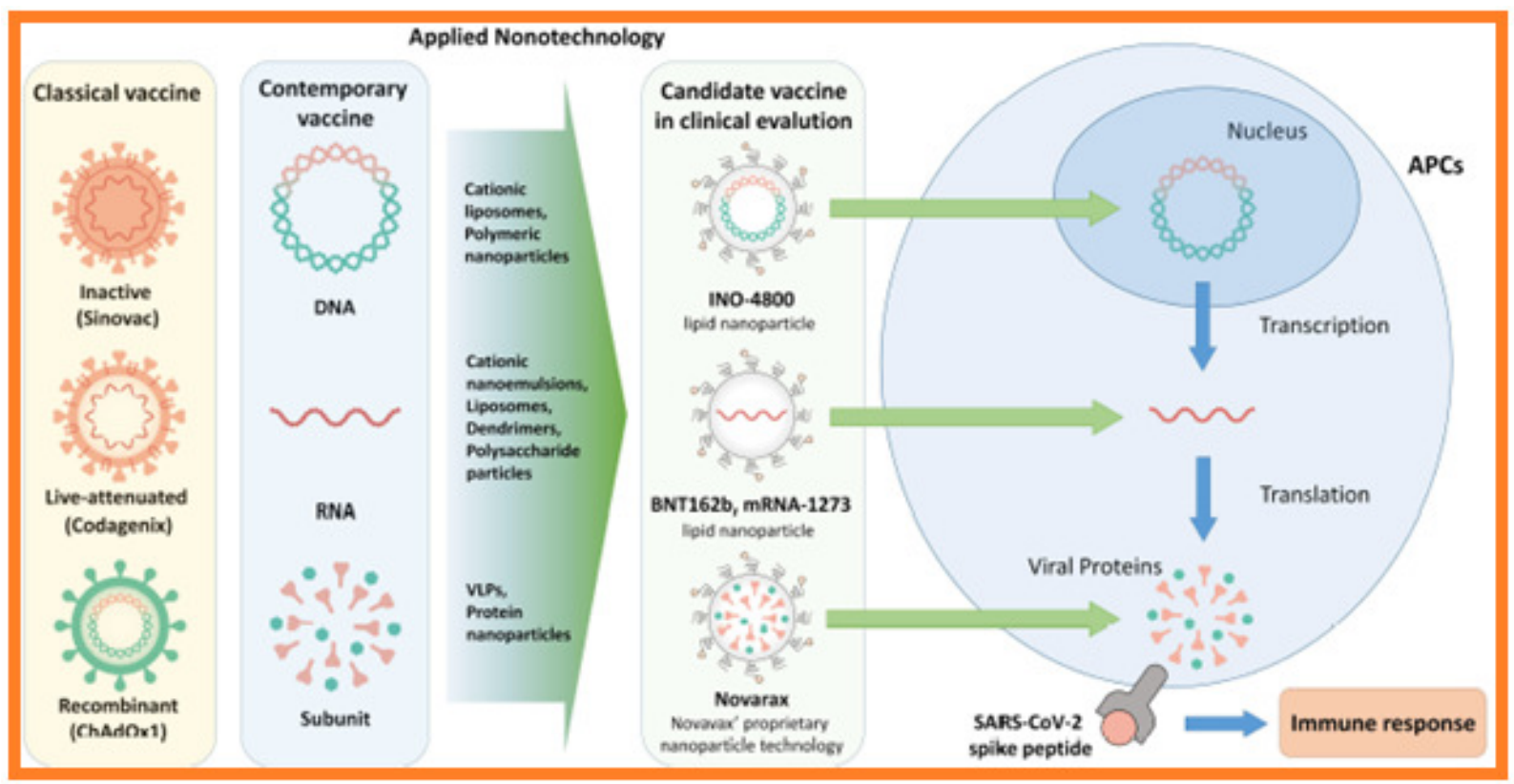

Figure 1. Classical vaccine, Moderna vaccine and nanotechnology applied vaccine against SARS-CoV-2 
In studies, nanoparticles similar to immunogenic viruses have been developed and produced with nanoparticle vaccine technology (Figure 1) [15]. Also developed by Inovio Pharmaceuticals, Inc., Plymouth Meeting, PA, USA, INO-4800 is a candidate DNA vaccine among nucleic acid vaccines (Figure 1). Similar to RNA vaccines, INO-4800 is a nucleic acid vaccine that can induce an immune response by being translated into proteins within human cells [16]. An RNA vaccine candidate against SARS-CoV-2 is now known as mRNA-1273 (Moderna, Cambridge, MA, USA) (Figure 1). This vaccine contains a synthetic mRNA strand so that the binding site for ACE2 can be translated into a pre-modified SARS-CoV-2 S protein [17]. BNT162b1, under development by Pfizer, New York, NY, USA, is an optimized mRNA vaccine with the codon encoding the SARS-CoV-2 RBD (Figure 1) [18].

\subsection{Micro Pumping}

The micropump has a pump inlet and an outlet. It can continuously create a vacuum or negative pressure at the inlet. At the outlet, large outlet pressure is created. It is a small-sized device whose working medium is liquid. In these microfluidic devices, silicone-based elastomeric materials based on the lithography technique called PDMS are used. PDMS is also a non-toxic, gas-permeable molecule to cells and excellent optically compatible. Microfluidic cell culture devices have pumps and valves that allow small volumes of liquid to be transferred [19]. In 1979, the first magnetic MEMS microvalve was developed. The function of the microvalve used in microfluidic systems is to change the flow direction at the desired time to control and regulate the liquid flow. In microfluidic systems, microvalves are the most important component of integrated LOC devices. These high-performance microvalves can perform sequential loading and washing operations accurately and quickly [20]. This feature is in high demand in the on-chip biomedical lab or point-of-care devices. It is a compact, robust, selfpowered micropump that does not need a complex external system. However, in today's microfluidic technologies there is a need to develop a micro-valve that can change fluid flow quickly, without leakage, and with less dead volume [21]. Magnetic micro-valves used today can often be hybrid integrated with permanent magnets to increase magnetic forces with less power consumption. Electromagnetic micro-valve representation is given in Figure 2.

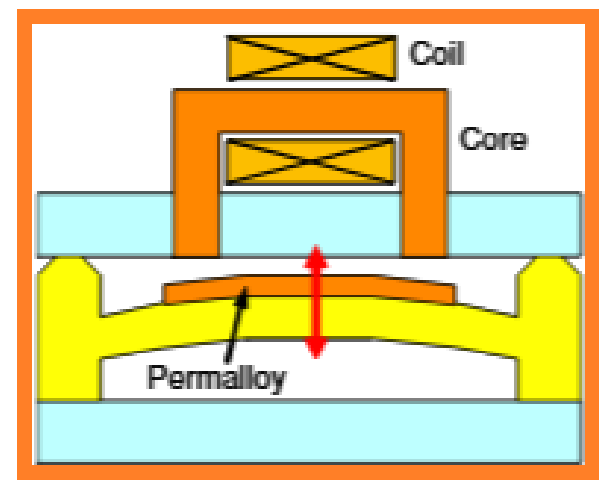

Figure 2. Micro valve and amplifier unit [22]

Various pumping methods such as finger-operated pumping, capillary pumping, gravity-based pumping and pre-degassed pumping have been developed to get rid of complex structures. Today, a vacuum-assisted pumping method using the gas permeability or solubility of PDMS is highly suitable for many biomedical microfluidic systems due to its simple application [23]. Wang et al. They have developed a compact, syringe-assisted, vacuum-powered micro-pump 
module that can be easily attached to the outlet of any existing microfluidic device and provides greater flexibility in many biomedical applications. They whether integrated and analyzed a polymeric microfluidic device into a portable mechanical micro-pumping system that uses liquid-sensitive polymer particles as an actuator without an external power supply. Xu et al. They are reported that the flow of water from the semipermeable membrane to the osmotic reagent reservoir facilitates the fluid flow in the channel by the osmosis process when a semipermeable membrane is sandwiched between the inner osmotic reagent reservoir and the external water reservoir [24]. Updates to osmotic driven passive pumping techniques in micro fluids are summarized in Table 1.

Table 1. Passive pumping techniques [25-26]

\begin{tabular}{|c|c|c|c|}
\hline \multicolumn{5}{|c|}{ Passive Pumping Techniques in Microfluidics } \\
\hline Materials Used & Flow Rate & Advantages & Disadvantages \\
\hline PDMS cubic chambers & $0.15 \mu 1 \mathrm{~h}^{-1}$ & $\begin{array}{c}\text { It provides the concentration } \\
\text { gradient for more than a week }\end{array}$ & Low flow rate \\
\hline PDMS and natural rubber & $17 \mu 1 \mathrm{~min}^{-1}$ & $\begin{array}{c}\text { It can be placed directly on a } \\
\text { microdevice }\end{array}$ & $\begin{array}{c}\text { Reactivation of pump } \\
\text { requires water }\end{array}$ \\
\hline PDMS chamber & $0.33 \mu \mathrm{min}^{-1}$ & $\begin{array}{c}\text { Low flow rate is used for constant } \\
\text { refreshing of culture medium }\end{array}$ & $\begin{array}{c}\text { Regular refreshing of } \\
\text { osmotic reagent }\end{array}$ \\
\hline
\end{tabular}

\section{MICROFLUIDIC SYSTEMS}

The first microfluidic devices were generally made of silicone and glass. Researchers used silicon and glass with the technology of that time. However, these products have some drawbacks. Silicone is expensive and cannot be combined in optical microscopy due to its opacity. Also, both silicone and glass have low gas permeability. Next, the researchers examine organic polymers as the most viable option to develop an alternative that can be optically transparent, easy to process, flexible and inexpensive compared to its predecessors. Later, PDMS was developed microfluidic systems in the 1990s. With this development, it is preferred as the most used material in microfluidics and is widely used today. Compared to traditional materials used in other micro electrics, one of the main advantages of PDMS is its compatibility with cells and the ease of culturing simple organisms. In the late 1990s, it was observed that microfluidic devices were created for cell biology applications such as cell and protein separation modeling, cell-based biosensors, culture and research [27]. Towards the 2000s, it is seen that researchers started to work on microfluidic devices that can be used as tissue and organ models for drug discovery and development. In these studies, they developed the (organson-a-chip) by examining pathophysiology and biological processes. Since the 2000s, many tissue models of gut, liver, brain, heart, eye, skin, lung, muscle, blood vessels and tumor have been studied on a chip [28]. In 2010, a biometric device was developed to simulate the lung alveolar-capillary interface in structural, functional and mechanical aspects. Thanks to this work, a functional micro-environment was created and integration of different tissues into a single chip was achieved. The developed device has human alveolar epithelial cells on one side and human pulmonary endothelial cells on the opposite or underside. Thus, it consists of two PDMS microchannels separated by a porous PDMS membrane forming epithelial and endothelial compartments. It mimics human breathing by injecting air into the sidewall or walls of the alveoli and then vacuuming it [29]. It appears that an organ chip study was conducted in the human intestine in 2018. One of the most important features of the human intestine is digestion and absorption. It is also where the gut microbiome and microbes live in common. In this study, drug absorption was attempted in an organ chip made with an intestinal model. 
Intestinal samples were placed in the upper part of the developed microfluidic portion, and a porous membrane separating it from the bottom was placed to check whether the administered drugs were absorbed. Precise control of the volume and manipulation of liquids is essential for many scientific fields, including chemistry, analytical biochemistry, biotechnology, and engineering. Microfluidics has become an important tool for designing environments with precise control of the conditions under study [30]. Microfluidics often includes devices and methods for controlling and manipulating liquids at a submillimeter scale. This technology has been presented as a popular candidate to replace traditional experimental approaches, especially in the biomedical field. The most popular and common approach for manufacturing microfluidic devices involves the use of "soft lithography" of poly-dimethylsiloxane (PDMS). This method has greatly contributed to the development of microfluidic technology. The use of these materials makes it easy to mold structures with micrometric resolution using a simple casting mold [31]. Different methods have been developed for the manipulation of both liquids and particles in microfluidics using electrical, magnetic, optical, capillary and mechanical forces [32]. It is seen that microfluidic devices are created by various integration of fluids, electronics, optics and biosensors. The main purpose is to meet the need for pathological analysis while the fluid is in motion. Microfluidic systems have proven useful in finding methods to diagnose fatal and chronic diseases at an early stage. A very-large-scale integration (VLSI) system is the process of creating integrated circuits by combining thousands of transistors on a single chip. With modern technologies, the number of transistors that can be installed per unit area has increased to billions, and this term has begun to give way to the term ULSI (Largest Scale Integration). Due to the emergence of advanced technologies such as MEMS, NEMS, it has become possible to integrate multiple interdisciplinary modules into a single-chip device. The interdisciplinary interaction of this technology can be schematized as shown in Figure 3.

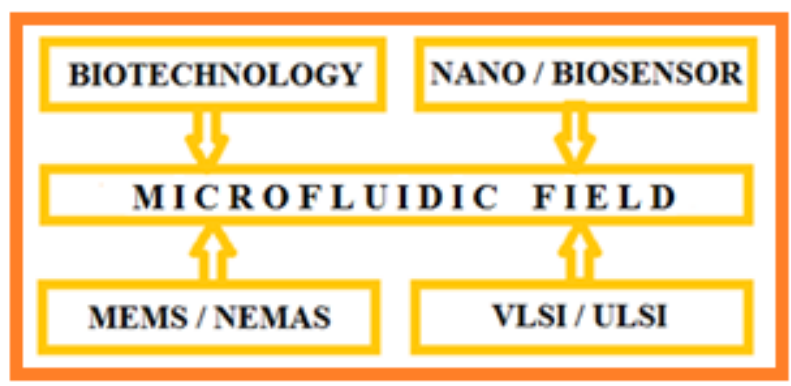

Figure 3. Interdisciplinary interaction in microfluidic technology

PDMS offers features such as low cost, optical transparency, flexibility, gas permeability, ease of use and high accuracy repeatability of models. The combination systems were created to allow both miniaturization and parallel matching of processes in compact devices, saving reagents and costs. Thanks to the easy production and flexibility of these devices, various functional microfluidic elements such as sensors, mixers, separators, distributors, pumps, valves have been produced. However, some concerns have arisen regarding the use of PDMS for biomedical research. It was found that leakage of non-crosslinked oligomers from PDMs can be toxic to cells. Due to its hydrophobic properties and permeability, it has been observed that hydrophobic small molecules are absorbed by PDMS. In addition, water vapor permeability can cause rapid evaporation, which can adversely affect experiments, mainly without static flow. However, the disadvantages associated with PDMS can be mitigated and additional device preparation may be required [33]. Recently, alternative materials to PDMS, namely thermoplastics (polystyrene - PS, cyclic olefin copolymer - COC, polymethyl methacrylate PMMA and polycarbonate - PC), paper, Wax and textile products have been investigated. 
Mukhopadhyay et al. They appear to suggest a microchannel bend in polymethylmethacrylate (PMMA) of different widths. The effects of channel aspect ratio and different separation angles were examined in terms of fluid flow. Mukhopadhyay et al. It suggests a microchannel bend in polymethylmethacrylate (PMMA) of different widths. The effects of channel aspect ratio and different separation angles in terms of fluid flow were investigated [34].

In connection with microfluidic technology, microfluid applications assume full integration of various microfluidic components and procedures in a single chip for miniaturizing chemical and biological processes [35]. Organ on a Chip refers to complex microengineering systems that aim to mimic the physiological basic properties of certain human organs, human tissues and interactions. There are several steps involved in the processing of the microfluidic device. Microfluidic processing begins with the collection of the physiological sample and then the specific analyte/biomarker is extracted from that sample. The transducer will act on the analyte electrically, electromechanically, optically or mechanically, depending on the biomedical application. The next step involves counting, classifying and raising the transducer output by the application. Finally, the amplified sample is processed using microelectronic methods. The latest trend shows that research in this area is increasing [36]. Many projects and research groups have been established in many countries of the world. The main purpose of these groups is to use micro/nanostructures; To develop new micro and nano technologies for microfluidics and to demonstrate new microfluidic applications in biomedical and life sciences.

\section{MICROFLUIDIC TECHNOLOGY APPLICATION}

The demand for microfluidic devices in many areas is due to the various technological advantages of fluid technology such as portability, automatic sample handling and reconfigurability. Real-time PCR detection chips for bacteria detection are some of the applications of microfluidic technology in the biomedical field, such as DNA chip, gene chip, cellular analysis chip, flow cytometry (for HIV) [37]. Wooseok et al. It appears that they focus on the properties of microfluidic chips to be used to meet the requirements of point-of-care diagnostic systems and increase their efficiency [38]. Since the functional modules and operating principles required in microfluidic systems are generally dependent on target analytes, applications of POCT systems (Point-of-care tests) are categorized by analyte types such as cells, proteins, metabolites and nucleic acids. POCT systems (point of care tests) are capable of detecting certain biomarkers from these analytes. These different biomarkers require different tests, diagnostic principles, and operating systems. Thus, in each category, the configuration of modules, detection methods, and advantages and disadvantages of microfluidic-based POCT diagnostic systems are reviewed. Cardoso et al. worked for the development of disposable and fully integrated microfluidic devices for microfluidic and clinical applications in biological fluids and monitoring of concurrent parameters [39]. This chip is produced by integrating biosensors, optical filters and electronic circuits on a single flow technique to promote pumping and mixing of microfluidics in microchannels. Mathematical models are required to complete the design of the channel. Therefore, Makhijani et al. developed a numerical model to simulate liquid filling due to the presence of surface tension at the liquid-air interface and demonstrated the application of disposable biochips for clinical diagnosis. It was mainly used for analysis and optimization to achieve the desired flow [40]. It appears that a transducer based on a piezoelectric material such as $\beta$-PVDF (polyvinylidene fluoride prepared in phase) is used to create this acoustic flow. This polymer is processed to be functionally graded to maintain heating and control the motion of fluids along with the input signal applied to the converter. To facilitate portability with less mixing time and to achieve high sensitivity and reliability in microfluidic systems, it is recommended to use a white light source and spectrophotometry as a sensing technique that can be made possible by combining 
highly selective optical interference filters. Katla Sai et al. discussed the impact of inorganic nanomaterials on biofunctionalization, synthesis and clinical translation assessment for biomedical applications of microfluidic devices [41]. Microfluidic methods result in less energy consumption, rapid synthesis of quantum dots, and inorganic nanomaterials such as metals, nanocomposites and metal oxides. This also demonstrates the need for LOC devices for faster completion of clinical translations by conducting superior in vitro studies. There are three main types of biochips: lab-on-chip (LOCs), DNA chips, and protein chips. LOCs use a combination of one or more lab functions in a single integrated chip [42]. Due to recent advances in computational simulations, it is now possible to synthesize nanomedicine applications based on inorganic nanomaterials into a single structure using microfluidic technology useful for therapeutic applications. Recent advances in microfluidic technology and design strategies lead to the development of useful universal sample-result microfluidic devices to effectively detect pathogens with high specificity and sensitivity [43]. There are goals to be designed to have many advantages, such as main focus, point-of-care diagnosis, processing of small volume samples, fast detection time, miniaturization, and portability. In addition, emphasis is placed on the development of accessory-free and fully integrated standalone microchips. These advances will help reduce mortality and control the spread of life-threatening diseases such as Tuberculosis (TB), HIV and the like. Wen et al. gave an overview of microparticles produced by droplet microfluidics in their compilation studies [44]. In addition, recent developments in the biomedical fields are discussed. In subsequent developments, the droplet formation mechanism was designed and devices used to create various droplets were described. Methods for preparing template microparticles from these droplets are summarized and the unique and complex structures provided by microfluidic techniques are highlighted. Next, the biomedical applications of these microparticles are explained with a focus on recent advances in their use as drug delivery devices and cell-loaded matrices. Other applications are also briefly described, including biosensors and artificial cells. Finally, the current challenges of these microparticles could potentially are discussed and concluded with perspectives and possible implications. Auerswald et al. Their studies reveal the need for the development of microfluidic devices necessary for the multiplex detection of various antibiotic families in raw milk [45]. It has been reported that four antibiotic families are overused in the dairy diet, leading to stronger bacterial resistance. This will pose a serious problem and threaten effective antibacterial therapy in humans. An automatic, easy-to-use, fast and cost-effective multiplex detection system has been developed to detect these antibiotic families. The design principle of the microreactor including microfluidic mixing reactor and ordinary micro reactor types is introduced [46]. Next, the latest advances in microreactor applications are reviewed (reaction kinetics, enzymatic bio reactions, biosynthesis and medical testing with the micro-mix reactor and rtPCR, ELISA immune reaction and nucleic acid hybridization with ordinary microreactor strains). Finally, on-chip detection methods such as common and low-cost methods (laser-induced fluorescence, ultraviolet (UV) absorption, and electrochemical method) are summarized. Kalaitzakis et al. It is seen that he explains the proteomic profiling techniques integrated with knowledge management methodologies and analysis platforms [47]. Its main purpose is to identify clinically relevant analytes and biomarkers for early-stage detection of pancreatic cancer. Ultrasonic and physical detection of pancreatic cancer becomes complex and burdensome as the pancreas is deep in the abdominal cavity. Approximately 95\% of such cases are diagnosed in the last stages; For example, stage III or IV causes a high mortality rate. The Loccandia project is primarily concerned with the diagnosis of early-stage cancer of the pancreas. This can be done through a microfluidic development, namely the validation of the plasma protein integrated with the application of profiling with the help of a new nanotechnology-based platform and a complete proteomic analysis chain. The success of this project is based on the seamless combination of bio, nano and data processing and knowledge management 
methodologies. Ziober et al. describe the emergence of microfluidic devices for biomarkerbased identification and early diagnosis of oral cancer [48]. It is seen that head and neck cancers constitute approximately $40 \%$ of oral cavity cancers. Oral cavity cancer includes squamous cell carcinoma that occurs on the lips, mouth, tongue, gums, buccal mucosa, hard and soft palate, and floor. OSCC (Oral squamous cell carcinoma) is deadly cancer and results in high mortality, morbidity, and deformity. Traditional diagnostic and screening methods for OSCC are not costeffective and highly accurate and require lengthy and detailed procedures by advanced equipment, modern laboratories, and qualified personnel. To overcome these problems, a miniature, accurate, automatic, integrated and inexpensive microfluidic chip is required. Screening patients for OSCC will be considered as this microfluidic saliva input sample, and then processed by minimally trained personnel, thus providing timely results. Identification of oral cancer and its precursor (starting chemicals used in the synthesis of a chemical substance) will be possible with the cells in the chip, the membrane-associated cell proteins expressed alone in the membranes of dysplastic and cancer cells and have unique gene transcription profiles. Dutse et al. studied the need for microfluidic-based chips that can be used as complementary tools to control the effects of pathogenic agents to prevent environmental damage [49]. These systems are easy to use, fast, precise, reliable enough and portable. Therefore, it offers many advantages over traditional methods that are expensive, tedious and time-consuming. The main application of microfluidic systems in pathogen detection includes DNA-based methods based on electrochemical techniques. Fluid mechanics of systems are discussed at the nanoscale. The main limitation is that the use of mechanical pumps required for liquid transfer requires attention and reduces performance. Three examples are presented to illustrate the potential of microfluidics in the medical field. First, when it comes to patients monitoring blood lithium, a pre-filled, disposable chip-based on capillary electrophoresis is discussed [50]. Orally, lithium is often used to treat patients suffering from bipolar disorder. Therefore, a glass chip based on vacuum pre-filled with capillary electrophoresis and buffer solution was produced. Vacuum refers to any area where the pressure is less than atmospheric pressure (negative pressure). Vacuum-operated devices utilize the ability of an MFD to absorb the sample through negative pressure without any extra on/off the microfluidic unit. However, in osmosis-induced Mfds, the osmotic reagent had to be refreshed at regular intervals, which limited their application [51]. LOC applications, such as pumping the fluid flow with the platforms reported by the valve, and separating and detecting the different chemical species applied in a microfluidic format, require a review of the modes of operation on different chips. Finally, microfluidic cell culture has been developed expressing the use of surface tension in passive pumping based on the physical and micro-related concept environment where differential pressure is generated due to different volumes at the inlet and outlet port to aid fluid flow [52]. The same chip has the potential to detect magnesium and calcium in cow blood to detect milk fever. It is seen that the sodium in the urine can also be measured with this chip platform, which is very useful for kidney patients. In the second example, a simple chip is presented that is used to count sperm cells in sperm to determine male fertility. Finally, improved in vitro models used for drug development are presented. As an example of the organ on-chip, the blood-brain barrier on the chip has been noticed to create a versatile platform for the screening of drugs and has the potential to greatly alter and reduce animal testing [53]. The need for simple, fast and timely diagnosis and elimination of global alarming diseases such as malaria has led to the emergence of microfluidic PCR diagnostic methods. Taylor et al. His work has identified a microfluidic technology that has the potential to overcome the costly, complex and challenges of traditional molecular diagnostics, especially in developing countries [54]. RT-PCR testing is a widely used and highly specific messenger RNA detection and quantification technique that can detect the presence of SARS-CoV-2 in a biological sample. Real-time RT-PCR testing uses fluorescent dyes to allow scientists to see results almost 
instantly [55]. Ai et al. reported that they have 97\% high sensitivity for detecting COVID-19 by CT in adults when used as the reference RT-PCR. This study also demonstrated how the timeline of Negative-Positive chest CT scans compares with the corresponding RT-PCR test timelines [56]. Although the RT-PCR test plays a very important role in accurately detecting SARS-CoV-2 on a case-by-case basis, it also has inherent problems that limit its usefulness. Current barriers to the widespread use of RT-PCR testing include the lack of test kits and a long processing time before results are obtained [57]. Microfluidic devices, the structure is given in Figure 4, allow the analysis of various samples such as blood, saliva or cell tissues to provide a fast and accurate diagnosis.

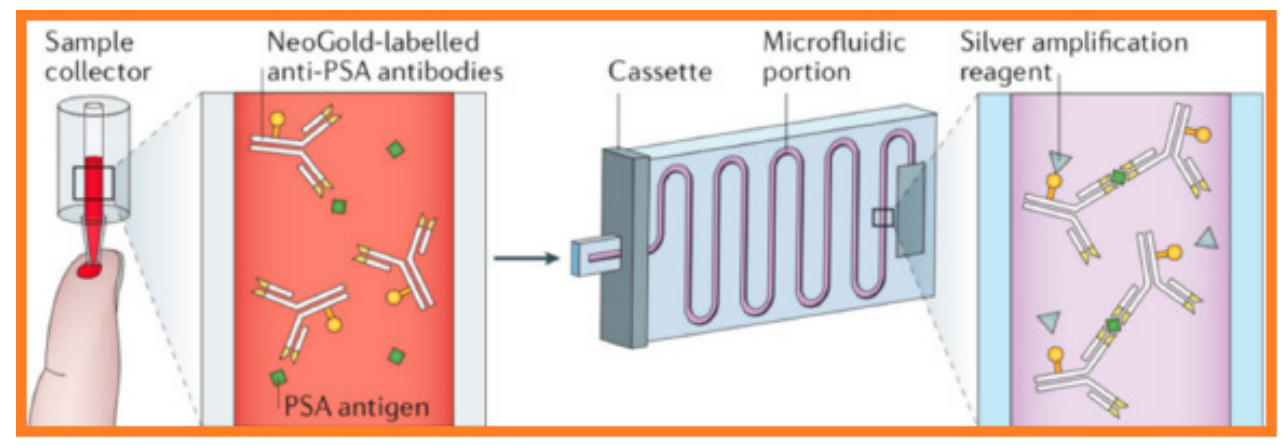

Figure 4. An example of a microfluidic diagnostic device [58]

Microfluidic platforms are created for microbial extraction and combined with a variety of analytical methods that detect pathogenic microorganisms [59]. For example, a microfluidic chip can catch pathogens in the air. Thus, the laminar flow is transformed into the curved airflow in the device, increasing the possibility of contact with the bacteria in the airflow with the duct wall. Microfluidic platforms can collect hundreds of bacteria in a few microliters of aqueous media, which is sufficient for direct immunoassay or nucleic acid analysis. This technique cannot work on its own, but it facilitates sampling and downstream bioanalysis [60].

\section{CONCLUSIONS}

Microfluidics is widely used in different fields such as biomedical, engineering and chemistry. Depending on the nature of the application, a person can only design and prototype a microfluidic device. The described micromixer, microvalve and micro motor are considered good candidates for use in existing microfluidic devices. According to studies conducted or to be conducted, microfluidic chip technology has a wide range of applications in the biomedical field. Its microfluidic devices are suitable for point-of-care diagnosis as they provide fast and timely diagnostic results. For many other applications, platforms have been created to design microfluidic devices.

The complex design of microfluidic chips, the stable binding and integration of different functional organ chips, and the modification and development of different culture conditions in various cell cultures require further optimization and experimentation. The problems of molecular absorption, mass transfer and bubble formation within the equipment require immediate solutions. Good operational stability and repeatability are essential for the introduction of the method. In addition, costly and complex preparation and operation processes are also issues to consider. If more convenient, user-friendly and inexpensive devices can be developed, the microfluidic chip promises to play a greater role in pharmaceutical analysis. 


\section{REFERENCES}

[1] Saggiomo, V. and Velders, A. H. (2015). Simple 3D printed scaffold-removal method for the fabrication of intricate microfluidic devices. Adv. Sci. 2, 1-5.

[2] Whitesides, G. (2006). The origins and the future of microfluidics. Nature 442, 368-373.

[3] Sackmann, E., Fulton, A. and Beebe, D. (2014). The present and future role of microfluidics in biomedical research. Nature 507, 181-189.

[4] Luo, T., Fan, L., Zhu, R., Sun, D. (2019). Microfluidic Single-Cell Manipulation and Analysis: Methods and Applications. Micromachines 10(2): 104.

[5] Suwannaphan, T., Srituravanich, W., Sailasuta, A., Piyaviriyakul, P., Bhanpattanakul, S., Jeamsaksiri, W., Sripumkhai, W., Pimpin, A. (2019). Investigation of Leukocyte Viability and Damage in Spiral Microchannel and Contraction-Expansion Array. Micromachines. 10(11): 772.

[6] Nielsen, J. B., Hanson, R. L., Almughamsi, H. M., Pang, C., Fish, T. R., Woolley, A. T. (2020). Microfluidics: Innovations in Materials and Their Fabrication and Functionalization. Anal. Chem. 92, 150-168.

[7] Young, E. W., Beebe, D. J. (2010). Fundamentals of microfluidic cell culture in controlled microenvironments. Chemical Society Reviews, 39(3): 1036-1048.

[8] Baydoun, M., Treizeibré, A., Follet, J., Vanneste, S. B., Creusy, C., Dercourt, L., Delaire, B., Mouray, A., Viscogliosi, E., Certad, G., Senez, V. (2020). Micromachines. 11(2): 150.

[9] Luo, Y., Zhao, J., He, C., Lu, Z., Lu, X. (2020). Miniaturized Platform for Individual Coral Polyps Culture and Monitoring. Micromachines. 11(2): 127.

[10] Williams, M. J., Lee, N. K., Mylott, J.A., Mazzola, N., Ahmed, A., Abhyankar, V. V. (2019). A Low-Cost, Rapidly Integrated Debubbler (RID) Module for Microfluidic Cell Culture Applications. Micromachines. 10(6): 360 .

[11] Horiuchi, T., Miura, T., Iwasaki, Y., Seyama, M., Inoue, S., Takahashi, J. İ., Haga, T., Tamechika, E. (2012). Passive Fluidic Chip Composed of Integrated Vertical Capillary Tubes Developed for On-Site SPR Immunoassay Analysis Targeting Real Samples. Sensors. 12(6): 7095-7108.

[12] Fu, J., Wu, L., Qiao, Y., Tu, J., Lu, Z. (2020). Microfluidic Systems Applied in Solid-State Nanopore Sensors. Micromachines. 11(3): 332.

[13] Chokkalingam, V., Tel, J., Wimmers, F., Liu, X., Semenov, S., Thiele, J., Figdor, C. G. and Huck, W. T. (2013). Probing cellular heterogeneity in cytokine-secreting immune cells using droplet-based microfluidics, Lab Chip, 13, 4740-4744.

[14] Sahu, K. K., Lal, A., Mishra, A. K. (2020). Latest updates on COVID-2019: a changing paradigm shift. J Med Virol. 92(6):533-535. doi:10.1002/jmv.25760

[15] Coleman, C. M., Liu, Y. V., Mu, H. Y., et al. (2014). Purified coronavirus spike protein nanoparticles induce coronavirus neutralizing antibodies in mice. Vaccine. 32(26): 3169-3174.

[16] Sheahan, T. P., Sims, A. C., Leist, S. R., et al. (2020). Comparative therapeutic efficacy of remdesivir and combination lopinavir, ritonavir, and interferon beta against MERS-CoV. Nat Commun. 11(1). doi:10.1038/s41467-019-13940-6

[17] Tu, Y. F., Chien, C. S., Yarmishyn, A. A., et al. (2020). A review of SARS-CoV- 2 and the ongoing clinical trials. Int J Mol Sci. 21(7): 2657. doi:10.3390/ijms210726572

[18] Mulligan, M. J., Lyke, K. E., Kitchin, N., et al. (2020). Phase 1/2 study of COVID-19 RNA vaccine BNT162b1 in adults. Nature. 586(7830):589-593. doi:10.1038/s41586-020-2639-4 
[19] Huh, D., Hamilton, G. A., and Ingber, D. E. (2011). From 3D cell culture to organs-on-chips. Trends in cell biology, 21(12): 745-754.

[20] Wang, A., Koh, D., Schneider, P., Breloff, E., Oh, K. W. (2019). A Compact, Syringe-Assisted, VacuumDriven Micropumping Device. Micromachines. 10(8): 543.

[21] Fu, C., Rummler, Z., Schomburg, W. (2003). Magnetically driven micro ball valves fabricated by multilayer adhesive film bonding," J Micromech Microengineering, vol. 13, pp 96-102.

[22] Goettsche, T., Kohnle, J., Willmann, M., Ernst, H., Spieth, S.,Tischler, R., Messner, S., Zengerle, R., Sandmaier, H., (2005). Novel approaches to particle tolerant valves for use in drug delivery systems Sensors Actuators 118, 70-7.

[23] Xu, Z. R., Yang, C. G., Liu, C. H., Zhou, Z., Fang, J. and Wang, J. H. (2010). An osmotic micro-pump integrated on a microfluidic chip for perfusion cell culture, Talanta, 80(3): 1088-1093.

[24] Park, J. Y., Kim, S. K., Woo, D. H., Lee, E. J., Kim, J. H. and Lee, S. H. (2009). Differentiation of Neural Progenitor Cells in a Microfluidic Chip-Generated Cytokine Gradient, Stem Cells, 27(11): 2646-2654.

[25] Paguirigan, A. L., and Beebe, D. J. (2009). From the cellular perspective: exploring differences in the cellular baseline in macroscale and microfluidic cultures, Integr. Biol. 1(2): 182-195.

[26] Folch, A. and Toner, M. (2008). Cellular micro models on biocompatible materials, Biotechnology Programs, skin. 14(3): 388-392.

[27] Huh, D., Matthews, B. D., Mammoto, A., Montoya-Zavala, M., Hsin, H. Y. and Ingber, D. E. (2010). Reconstruction of lung function at the organ level on a chip, Science, 328(5986): 1662-1668.

[28] Bae, B., Kim, N., Kee, H., Kim, S., Lee, Y., Lee, S., Park, K. (2002). Feasibility test of an electromagnetically driven valve actuator for glaucoma treatment, J Microelectromech Syst, vol. 11, pp. 344-354.

[29] Esch, E. W., Bahinski, A. and Huh, D. (2015). Organs on chips at the borders of drug discovery, Nature Reviews Drug Discovery, skin. 14(4): 248-260.

[30] Hou, X., Zhang, Y. S., Trujillo-de Santiago, G., Alvarez, M. M., Ribas, J., Jonas, S. J. and Khademhosseini, A. (2017). Interplay between materials and microfluidics. Nature Reviews Materials, 2(5): 1-15.

[31] Bhattacharjee, N., Urrios, A., Kang, S., and Folch, A. (2016). The upcoming 3D-printing revolution in microfluidics. Lab on a Chip, 16(10): 1720-1742.

[32] Trantidou, T., Elani, Y., Parsons, E., and Ces, O. (2017). Hydrophilic surface modification of PDMS for droplet microfluidics using a simple, quick, and robust method via PVA deposition. Microsystems \& nanoengineering, 3(1): 1-9.

[33] Pan, L. J., Tu, J. W., Ma, H. T., Yang, Y. J., Tian, Z. Q., Pang, D. W., Zhang, Z.L. (2018). Controllable synthesis of nanocrystals in droplet reactors. Lab. Chip. 18, 41-56.

[34] Mukhopadhyay, S., Roy, S. S., Mathur, A., Tweedie, M. and McLaughlin, J. A. (2010). Experimental study on capillary flow through polymer microchannel bends for microfluidic applications, Micromech. Microeng. 20(5): 055018.

[35] Samiei, E., Tabrizian, M., \& Hoorfar, M. (2016). A review of digital microfluidics as portable platforms for lab-on a-chip applications. Lab on a Chip. 16(13): 2376-2396.

[36] Li, W., Zhang, L., Ge, X., Xu, B., Zhang, W., Qu, L and Weitz, D. A. (2018). Microfluidic fabrication of microparticles for biomedical applications. Chemical Society Reviews, 47(15): 5646-5683.

[37] Mac Connell, A. B., Price, A. K. and Paegel, B. M. (2017). An integrated microfluidic processor for DNAencoded combinatorial library functional screening. ACS combinatorial science, 19(3): 181-192. 
[38] Wooseok, J., Han, J., Choi, J-W. and Ahn, C. H. (2015). Pointof-care testing (POCT) diagnostic systems using microfluidic lab-on-achip technologies, Microelectronic Engineering Journal, 132, 46-57.

[39] Cardoso, V. F., Catarino, S. O., Lanceros-Mendez, S., \& Minas, G. (2011). Lab-on-a-chip using acoustic streaming for mixing and pumping fluids. In 1st Portuguese Biomedical Engineering Meeting (1-4). IEEE.

[40] Makhijani, V. B., Reich, A. J., Puntambekar, A., Hong, C. and Ahn, C. (2001). Advances in passively driven microfluidics and lab-on-chip devices: a comprehensive literature review and patent analysis, Tech Connect Briefs, 1, 266-269.

[41] Krishna, K. S., Yuehao, L. i., Shuning, L. i. and Challa, S., Kumar, S. R. (2013). Labon-a-chip synthesis of inorganic nanomaterials and quantum dots for biomedical applications, Advanced Drug Delivery Reviews Journal, 65(11): 1470-1495.

[42] Volpatti, L. R. and Yetisen, A. K. (2014). Commercialization of microfluidic devices, Trends Biotechnol., 32(7): 347-350.

[43] Luo, G., Du, L., Wang, Y., and Wang, K. (2019). Recent developments in microfluidic device-based preparation, functionalization, and manipulation of nano-and micro-materials. Particuology, 45, 1-19.

[44] Li, W., Zhang, L., Ge, X., Xu, B., Zhang, W., Qu, L. and Weitz, D. A. (2018). Microfluidic fabrication of microparticles for biomedical applications. Chemical Society Reviews, 47(15): 5646-5683.

[45] Auerswald, J., Berchtold, S., Diserens, J. M., Gijs, M. A., Jin, Y. H., Knapp, H. F. and Voirin, G. (2009). Lab-on-a-chip for Analysis and Diagnostics: Application to Multiplexed Detection of Antibiotics in Milk. In Nanosystems design and technology (117-142). Springer, Boston, MA.

[46] Shi, H., Nie, K., Dong, B., Long, M., Xu, H. and Liu, Z. (2019). Recent progress of microfluidic reactors for biomedical applications. Chemical Engineering Journal, 361, 635-650.

[47] Kalaitzakis, M., Kritsotakis, V., Grangeat, P., Paulus, C., Gerfault, L., Perez, M., Reina, C., Potamias, G., Tsiknakis, M., Kafetzopoulos, D. and Binz, P. A. (2008). Proteomic based identification of cancer biomarkers: The LOCCANDIA integrated platform, Proceedings of 8th IEEE International Conference on BioInformatics and BioEngineering, Athens, 1-7.

[48] Ziober, B. L., Mauk, M. G., Falls, E. M., Chen, Z., Ziober A.F. and Haim H. B. (2008). Lab-on-a-chip for oral cancer screening and diagnosis, Head \& Neck Journal. 30(1): 111-121.

[49] Sabo, W. D., and Nor, A. Y. (2011). Microfluidics-based lab-on-chip systems in DNA-based biosensing: An overview, Sensors Journal, 11(6): 5754-5768.

[50] Aryasomayajula, A., Bayat, P., Rezai, P. and Selvaganapathy, P. R. (2017). Microfluidic Devices and Their Applications. In Springer Handbook of Nanotechnology (487-536). Springer, Berlin, Heidelberg.

[51] Xu, Z. R., Yang, C. G., Liu, C. H., Zhou, Z., Fang, J. and Wang, J. H. (2010). An osmotic micro-pump integrated on a microfluidic chip for perfusion cell culture, Talanta, 80(3): 1088-1093.

[52] Young, E. W. and Beebe, D. J. (2010). Fundamentals of microfluidic cell culture in controlled microenvironments, Chem. Soc. Rev., 39, 1036-1048.

[53] Bruijns, B., Van Asten, A., Tiggelaar, R., and Gardeniers, H. (2016). Microfluidic devices for forensic DNA analysis: A review. Biosensors, 6(3): 41.

[54] Taylor, B. J., Howell, A., Martin, K. A., Manage, D. P., Gordy, W., Campbell, S. D., \& Atrazhev, A. (2014). A lab-on-chip for malaria diagnosis and surveillance. Malaria Journal, 13(1): 179.

[55] Long, C., Xu, H., Shen Q, et al. (2020). Diagnosis of the coronavirus disease (COVID-19): rRT-PCR or CT? Eur J Radiol.126:108961 
[56] Ai, T., Yang, Z., Hou, H, et al. (2020). Correlation of chest CT and RT-PCR testing in coronavirus disease 2019 (COVID-19) in China: a report of 1014 cases. Radiology, 296(2): 41-45.

[57] Website. Chinese clinical guidance for COVID-19 pneumonia diagnosis and treatment, (2021). https://www.acc.org/latest-in-cardiology/articles/2020/03/17/11/22/chinese-clinical-guidance-for-covid-19pneumonia-diagnosis-and-treatment

[58] Mejía-Salazar, J. R., Rodrigues Cruz, K., Materón Vásques, E. M., Novais de Oliveira Jr, O. (2020). Microfluidic Point-of-Care Devices: New Trends and Future Prospects for eHealth Diagnostics. Sensors, 20, 1951.

[59] Zhang, D., Bi, H., Liu, B., Qiao, L. (2018). Detection of Pathogenic Microorganisms by Microfluidics Based Analytical Methods. Anal. Chem. 90, 5512-5520.

[60] Narimani, R., Azizi, M., Esmaeili, M., Rasta, S. H., Khosroshahi, H. T. (2020). An optimal method for measuring biomarkers: Colorimetric optical image processing for determination of creatinine concentration using silver nanoparticles. 3 Biotech. 10, 416. 